Pure and Applied Mathematics Quarterly

Volume 1, Number 3

(Special Issue: In Memory of

Armand Borel, Part 2 of 3 )

$683-699,2005$

\title{
Infinite Dimensional Groups and Automorphic $L$-Functions
}

\author{
Freydoon Shahidi
}

\section{INTRODUCTION}

In this note we will speculate on the possibility of establishing higher symmetric power transfers for cusp forms on $G L_{2}\left(\mathbb{A}_{F}\right)$, where $\mathbb{A}_{F}$ is the ring of adeles of a number field $F$, by means of extending the Langlands-Shahidi method to infinite dimensional groups (Section 6). It appears that a straightforward generalization $[14,15]$ of the theory of Eisenstein series from the finite dimensional theory $[39,40]$ may not suffice. A conceptually different generalization would be necessary if one wants to establish further transfers from forms on $G L_{m}\left(\mathbb{A}_{F}\right) \times G L_{n}\left(\mathbb{A}_{F}\right)$ to $G L_{m n}\left(\mathbb{A}_{F}\right)$, from which functoriality for higher symmetric powers could be obtained. We note that to obtain the fifth symmetric power transfer of a cusp form $\pi$ on $G L_{2}\left(\mathbb{A}_{F}\right)$, it would be enough to prove that $\pi \times \mathrm{Sym}^{4} \pi$ can be transferred to an automorphic representation $\pi \otimes \operatorname{Sym}^{4} \pi$ of $G L_{10}\left(\mathbb{A}_{F}\right)$, from which the existence of $\mathrm{Sym}^{5} \pi$ follows by an appeal to the classification of automorphic forms on $G L_{N}\left(\mathbb{A}_{F}\right)$. This was successfully accomplished for the transfer of forms on $G L_{2}\left(\mathbb{A}_{F}\right) \times G L_{3}\left(\mathbb{A}_{F}\right)$ to $G L_{6}\left(\mathbb{A}_{F}\right)$ in [34]. No higher transfers of forms on products of general linear groups is available at present. We refer to [44] for $G L_{2} \times G L_{2}$. We invite the reader to read Section 6 carefully to appreciate different aspects of this observation and evidence for it as well as its positive consequences towards Garland's work [14, 15] on establishing meromorphic continuation of Eisenstein series for loop groups.

We motivate the paper by discussing some very important conjectures in number theory such as those of Ramanujan-Petersson and Selberg on cuspidal representations of $G L_{2}\left(\mathbb{A}_{F}\right)$, as well as generic forms on groups whose connected $L$-groups have a classical derived group. We concentrate on the former and refer the reader to [56] for a detailed expository treatment of latter. We conclude

Received June 2, 2005.

Partially supported by NSF Grant DMS-0200325. 
the paper by recording the absolute convergence of $m$-th symmetric power $L$ functions for cusp forms on $G L_{2}\left(\mathbb{A}_{F}\right)$ for $\operatorname{Re}(s)>1$ and $m \leq 8$ which was left out from [33] (Theorem 7.2).

The paper is based on a talk given by the author at the International Conference in Memory of Armand Borel at the Center of Mathematical Sciences in Zhejiang University in 2004 for which I like to thank the organizers.

\section{Ramanujan Conjecture for Quasisplit Groups}

This is a quick summary of parts of [56].

Let $F$ denote either a number field or a function field and let $\mathbb{A}_{F}$ be its ring of adeles. Given a place $v$ of $F$, we let $F_{v}$ be the corresponding completion. Next, we let $\mathbf{G}$ be a connected reductive algebraic group over $F$ and set $G=\mathbf{G}\left(\mathbb{A}_{F}\right)$ and $G_{v}=\mathbf{G}\left(F_{v}\right)$. If $\mathbf{Z}$ is the center of $\mathbf{G}$, we then let $Z_{v}=\mathbf{Z}\left(F_{v}\right)$. We now fix a cuspidal (unitary) representation $\pi=\otimes_{v} \pi_{v}$ of $G$. Until counter examples were provided for either of the groups $S p_{4}$ or $U(2,1)$ (cf. $[23,35]$ ), it was believed that [46]:

(2.1) Ramanujan Conjecture: $\pi$ is tempered, i.e., each $\pi_{v}$ is tempered which simply means that all the matrix coefficients of $\pi_{v}$ are in $L^{2+\varepsilon}\left(Z_{v} \backslash G_{v}\right)$ for all $\varepsilon>0$.

As we just mentioned this is false in general. However, it is widely believed to be true for $G L(n)$.

When $\mathbf{G}$ is quasisplit, i.e., has a Borel subgroup $\mathbf{B}=\mathbf{T} \mathbf{U}$, defined over $F$, then one can consider a generic character $\psi$ of $\mathbf{U}(F) \backslash \mathbf{U}\left(\mathbb{A}_{F}\right)$, i.e., one which is non-trivial on the $\mathbb{A}_{F}$-points of every simple root subgroup of $\mathbf{U}$. A cuspidal representation $\pi=\otimes_{v} \pi_{v}$ of $G$ is called globally generic if there exists a function $\varphi$ in the space of $\pi$ such that

$$
\int_{\mathbf{U}(F) \backslash \mathbf{U}\left(\mathbb{A}_{F}\right)} \varphi(u g) \bar{\psi}(u) d u \neq 0 .
$$

The following version of Ramanujan Conjecture is expected to be true (cf. [43, $51])$.

(2.2) Conjecture. Assume $\mathbf{G}$ is quasisplit and $\pi$ is globally generic. Then $\pi$ is tempered, i.e., Ramanujan Conjecture is valid for globally generic cuspidal representations of quasisplit groups.

When $\mathbf{G}=G L_{n}$, every cuspidal representation is globally generic [57] and therefore our conjecture agrees with the general belief of the validity of the Ramanujan conjecture for $G L_{n}$.

The conjecture is unresolved even for $G L_{2}$ which we shall now elaborate. This will be the main theme of the present paper. We refer to [56] for a discussion 
of the conjecture for more general groups and to $[3,4,8,9,30]$ for the original papers.

\section{MaAss Forms and the Ramanujan Conjecture}

Let $\Gamma=\Gamma_{0}(N)$ be the Hecke subgroup of $S L_{2}(\mathbb{Z})$ of level $N, N \in \mathbb{N}$, i.e., if $\gamma=\left(\begin{array}{ll}a & b \\ c d\end{array}\right) \in \Gamma_{0}(N)$, then $c \equiv 0(N)$. Denote by $\mathfrak{h}$ the upper half plane and let $\Gamma \backslash \mathfrak{h}$ be the hyperbolic Riemann surface obtained by $\Gamma$ acting on $\mathfrak{h}$ through fractional linear transformations. Let $f \in L^{2}(\Gamma \backslash \mathfrak{h})$ be a normalized cuspidal eigenfunction for all the Hecke operator as well as $\Delta=-y^{2}\left(\partial^{2} / \partial x^{2}+\partial^{2} / \partial y^{2}\right)$. Write $\Delta f=s(1-s) f, s \in \mathbb{C}$; then

$$
f(x+i y)=\sum_{n \neq 0} a_{n}(|n| y)^{1 / 2} K_{s-1 / 2}(2 \pi|n| y) e^{2 \pi i n x},
$$

$a_{n} \in \mathbb{C}$, where for each $\nu \in \mathbb{C}, K_{\nu}(z)$ is the Whittaker-Bessel function bounded at infinity, i.e., the solution of

$$
t^{2} K_{\nu}^{\prime \prime}+t K_{\nu}^{\prime}-\left(t^{2}+\nu^{2}\right) K_{\nu}=0
$$

satisfying

$$
K_{\nu}(t) \sim \sqrt{\frac{\pi}{2 t}} e^{-t}
$$

as $t$ goes to $+\infty$.

Let $\lambda_{1}=\lambda_{1}(\Gamma \backslash \mathfrak{h})$ denote the smallest positive eigenvalue of $\Delta$ on $L^{2}(\Gamma \backslash \mathfrak{h})$.

(3.1) Selberg's conjecture [45, 47, 54]. $\lambda_{1} \geq 1 / 4$.

(3.2) Ramanujan's conjecture $[45,54] .\left|a_{p}\right| \leq 2 p^{-1 / 2}$.

Neither conjectures are proved so far. The best estimates towards both conjectures are due to Kim-Sarnak [32]:

$$
\begin{aligned}
& \left|a_{p}\right| \leq p^{-1 / 2}\left(p^{7 / 64}+p^{-7 / 64}\right) \\
& \lambda_{1} \geq \frac{1}{4}-\left(\frac{7}{64}\right)^{2}=0.2380371 .
\end{aligned}
$$

Conjecture (3.1) may be considered as the archimedean analogue of (3.2) and they are both instances of conjecture (2.2) as we explain below.

It is well-known (cf. [17]) that if $f$ as above is a new form with nebentypus $\omega$, then $f$ determines a unique irreducible constituent $\pi=\pi_{f}$ of

$$
L^{2}\left(G L_{2}(\mathbb{Q}) \backslash G L_{2}\left(\mathbb{A}_{\mathbb{Q}}\right), \omega\right) .
$$

Write $\pi=\otimes_{p} \pi_{p}$, where each $\pi_{p}$ is an irreducible unitary representation of $G L_{2}\left(\mathbb{Q}_{p}\right)$, where we understand that $\mathbb{Q}_{\infty}=\mathbb{R}$. Then (3.1) is equivalent to $\pi_{\infty}$ 
being tempered, while (3.2) gives the temperedness for all $p<\infty$. Thus (3.1) together with (3.2) is equivalent to Conjecture $(2.2)$ for $G L_{2}\left(\mathbb{A}_{\mathbb{Q}}\right)$.

It is better to take forms over an arbitrary number field $F$. One is then interested in irreducible constituents $\pi$ of $L^{2}\left(G L_{2}(F) \backslash G L_{2}\left(\mathbb{A}_{F}\right), \omega\right)$ of $L^{2}$-functions transforming under translation by elements $z \in \mathbb{A}_{F}^{*}$ of the center of $G L_{2}\left(\mathbb{A}_{F}\right)$ by $\omega(z)$, where $\omega$ is an idele class character of $\mathbb{A}_{F}^{*}$. We will, more generally, consider irreducible cuspidal constituents $\pi$ of $L^{2}\left(G L_{n}(F) \backslash G L_{n}\left(\mathbb{A}_{F}\right), \omega\right)$ for arbitrary $n$ and with similar meanings for $\omega$, etc. Then $\pi=\otimes_{v} \pi_{v}$ and for almost all $v<\infty$, class of $\pi_{v}$ is parametrized by a semisimple conjugacy class in $G L_{n}(\mathbb{C})$, or for simplicity, by a diagonal element $t_{v} \in G L_{n}(\mathbb{C})$ (cf. [5]).

When $n=2$, we can write

$$
t_{v}=\left(\begin{array}{cc}
\alpha_{v} & 0 \\
0 & \beta_{v}
\end{array}\right)
$$

$\alpha_{v}, \beta_{v} \in \mathbb{C}^{*}$. Then the Ramanujan Conjecture for $\pi$ simply requires

$$
\left|\alpha_{v}\right|=\left|\beta_{v}\right|=1
$$

for all such $v$.

The estimate (3.3) follows from

$$
p^{-7 / 64} \leq\left|\alpha_{p}\right|,\left|\beta_{p}\right| \leq p^{7 / 64}
$$

When $F$ is strictly larger than $\mathbb{Q},(3.5)$ is not available. In fact, for arbitrary $F$ one must contend to the slightly weaker result established by Kim-Shahidi in [33]

$$
q_{v}^{-1 / 9}<\left|\alpha_{v}\right|,\left|\beta_{v}\right|<q_{v}^{1 / 9} .
$$

A similar estimate based on $1 / 9$ can be established at archimedean places (cf. [29]), replacing (3.4).

For arbitrary $n$ and $F$, Luo, Rudnick and Sarnak have established the estimate

$$
q_{v}^{-\left(\frac{1}{2}-\frac{1}{n^{2}+1}\right)} \leq\left|\alpha_{i v}\right| \leq q_{v}^{\frac{1}{2}-\frac{1}{n^{2}+1}}
$$

in [42] which also holds at archimedean places. Here $t_{v}=\operatorname{diag}\left(\alpha_{1 v}, \ldots, \alpha_{n v}\right) \in$ $G L_{n}(\mathbb{C})$.

\section{Functoriality and Symmetric Powers}

The recent progress made towards the Ramanujan and Selberg conjectures relies on establishing certain new cases of Langlands functoriality conjecture [1, $28,33,34,38]$. In this section we will explain the case of symmetric powers for $G L_{2}$. 
Given an integer $m$, let $\mathrm{Sym}^{m}$ denote the $m$-th symmetric power representation of $G L_{2}(\mathbb{C})$ on symmetric tensors of rank $m$, or the homomorphism

$$
\operatorname{Sym}^{m}: G L_{2}(\mathbb{C}) \rightarrow G L_{m+1}(\mathbb{C})
$$

which is obtained as the matrix $\operatorname{Sym}^{m}(g)$ of change of coefficients of a homogeneous polynomial of degree $m$ under the change of variables

$$
(x, y) \mapsto(x, y) \cdot g .
$$

Let $\pi=\otimes_{v} \pi_{v}$ be a cuspidal representation of $G L_{2}\left(\mathbb{A}_{F}\right)$. Given an unramified place $v$, let $t_{v} \in G L_{2}(\mathbb{C})$ denote the corresponding diagonal element determining class of $\pi_{v}$. Then $\operatorname{Sym}^{m}\left(t_{v}\right) \in G L_{m+1}(\mathbb{C})$ will determine an unramified (spherical) representation of $G L_{m+1}\left(F_{v}\right)$ which we denote by $\operatorname{Sym}^{m} \pi_{v}$. More precisely, let

$$
\phi_{v}: W_{F_{v}}^{\prime} \rightarrow G L_{2}(\mathbb{C})
$$

be the two dimensional representation of the Deligne-Weil group $W_{F_{v}}^{\prime}$ parametrizing $\pi_{v}$ for all $v$ by Kutzko [36] and Langlands [41]. Then $\mathrm{Sym}^{m} \phi_{v}$ determines a $(m+1)$-dimensional representation of $W_{F_{v}}^{\prime}$. Let $\mathrm{Sym}^{m} \pi_{v}$ be the irreducible admissible representation of $G L_{m+1}\left(F_{v}\right)$ attached to $\operatorname{Sym}^{m} \phi_{v}$ by Langlands [41], Harris-Taylor [20] and Henniart [21]. When $\pi_{v}$ is spherical, this will be the same spherical representation we discussed earlier. Set

$$
\operatorname{Sym}^{m} \pi=\otimes_{v} \operatorname{Sym}^{m} \pi_{v} .
$$

(4.2) Conjecture (Langlands functoriality conjecture for symmetric powers). $\mathrm{Sym}^{m} \pi$ is an automorphic representation of $G L_{m+1}\left(\mathbb{A}_{F}\right)$.

(4.3) Theorem. $\mathrm{Sym}^{m} \pi$ is automorphic in the following cases:

1) $m=2$ (Gelbart-Jacquet [18])

2) $m=3$ (Kim-Shahidi [34])

3) $m=4(\operatorname{Kim}[28])$

While the automorphy of $\mathrm{Sym}^{2} \pi$ was established in 1978, the cases of $\mathrm{Sym}^{3} \pi$ and $\mathrm{Sym}^{4} \pi$ were not obtained until 2002.

Estimates (3.3), (3.4) and (3.5) are obtained by applying techniques of analytic number theory to the properties of $L\left(s, \mathrm{Sym}^{4} \pi, \mathrm{Sym}^{2}\right)$ and particularly its absolute convergence for $\operatorname{Re}(s)>1$ (cf. [6, 12, 25, 32, 33] and Corollary 7.4 here).

Estimate (3.6), proved in [33], and its archimedean counterpart, proved in [29], are obtained by applying a general result of [51, 52], Lemma 5.8, to the case $E_{8}-2$ of [51], with the representation of $\mathbf{M}\left(\mathbb{A}_{F}\right)$, tailored from $\mathrm{Sym}^{3} \pi$ and $\mathrm{Sym}^{4} \pi$ as explained in [33]. Observe that the derived group of $\mathbf{M}$ is isomorphic to $S L_{4} \times S L_{5}$. We refer to [55] for a survey of these results, as well as many other consequences of the existence of $\mathrm{Sym}^{3} \pi$ and $\mathrm{Sym}^{4} \pi$. 


\section{Some Triple Product L-Functions; More Functoriality}

The existence of symmetric cube is a consequence of another case of functoriality, namely, let

$$
\rho_{2} \otimes \rho_{3}: G L_{2}(\mathbb{C}) \times G L_{3}(\mathbb{C}) \rightarrow G L_{6}(\mathbb{C})
$$

be defined by sending

$$
\left(g_{1}, g_{2}\right) \mapsto g_{1} \otimes g_{2} .
$$

Langlands functoriality conjecture then requires that $\rho_{2} \otimes \rho_{3}$ be functorial. More precisely, let $\pi_{i}=\otimes_{v} \pi_{i v}$ be cuspidal representations of $G L_{i+1}\left(\mathbb{A}_{F}\right)$ for $i=1,2$. Let $\varphi_{i v}: W_{F_{v}}^{\prime} \rightarrow G L_{i+1}(\mathbb{C}), i=1,2$, be the 2- and 3-dimensional representations of $W_{F_{v}}^{\prime}$ parametrizing $\pi_{1 v}$ and $\pi_{2 v}$, respectively $([22,36,41])$. By the recent results of Harris-Taylor [20] and Henniart [21], as well as Langlands [41], one can attach an irreducible admissible representation $\pi_{1 v} \otimes \pi_{2 v}$ of $G L_{6}\left(F_{v}\right)$ to the six dimensional representation $\varphi_{1 v} \otimes \varphi_{2 v}$ of $W_{F_{v}}^{\prime}$. We set

$$
\pi_{1} \otimes \pi_{2}=\otimes_{v}\left(\pi_{1 v} \otimes \pi_{2 v}\right) .
$$

(5.1) Theorem (Kim-Shahidi [34]). The representation $\pi_{1} \otimes \pi_{2}$ is an automorphic representation of $G L_{6}\left(\mathbb{A}_{F}\right)$, i.e., $\rho_{2} \otimes \rho_{3}$ is functorial.

(5.2) Corollary [34]. $\mathrm{Sym}^{3} \pi$ is automorphic.

Corollary (5.2) is a consequence of the decomposition

$$
\pi \otimes \mathrm{Sym}^{2} \pi=\mathrm{Sym}^{3} \pi \boxplus\left(\pi \otimes \omega_{\pi}\right)
$$

of the automorphic representation $\pi \otimes \operatorname{Sym}^{2} \pi$. In fact, using $L$-functions and the classification theorem of Jacquet and Shalika [25], one can show that either

$$
\pi \otimes \mathrm{Sym}^{2} \pi=\pi_{1} \boxplus\left(\pi \otimes \omega_{\pi}\right),
$$

where $\pi_{1}$ is a cuspidal representation of $G L_{3}\left(\mathbb{A}_{F}\right)$, or

$$
\pi \otimes \mathrm{Sym}^{2} \pi=\left(\pi \otimes \omega_{\pi}\right) \boxplus\left(\pi \otimes \omega_{\pi} \eta\right) \boxplus\left(\pi \otimes \omega_{\pi} \eta^{2}\right)
$$

which happens if and only if $\mathrm{Sym}^{2} \pi \cong \mathrm{Sym}^{2} \pi \otimes \eta$ for a non-trivial cubic grössencha -racter $\eta$. We shall assume $\pi$ is not monomial. It then follows that either $\pi_{1}=\operatorname{Sym}^{3} \pi$ or $\left(\pi \otimes \omega_{\pi} \eta\right) \boxplus\left(\pi \otimes \omega_{\pi} \eta^{2}\right)=\operatorname{Sym}^{3} \pi$, proving the automorphy of $\mathrm{Sym}^{3} \pi$. (See Remark 5.9 at the end of this section.)

Theorem 5.1 is proved by applying a version of converse theorems of Cogdell and Piatetski-Shapiro $[10,11]$ to certain triple product $L$-functions $L\left(s,\left(\pi_{1} \otimes\right.\right.$ $\left.\left.\pi_{2}\right) \times \sigma\right)$ whose analytic properties are obtained from the Langlands-Shahidi method [19, 27, 48, 49, 50, 51, 52].

Let $S$ be a non-empty finite set of finite places such that for every $v \notin S$, $v<\infty, \pi_{1 v}$ and $\pi_{2 v}$ are both unramified. For each $n \in \mathbb{N}$, let $\mathcal{T}_{n}(S)$ denote the 
set of cuspidal representations of $G L_{n}\left(\mathbb{A}_{F}\right)$ which are unramified for all $v \in S$. Let $\mu$ be an idele class character of $\mathbb{A}_{F}^{*}$, highly ramified at one place in $S$. Let

$$
\mathcal{T}_{n}(\mu, S)=\mathcal{T}_{n}(S) \otimes \mu .
$$

To prove Theorem 5.1 we would need to consider $\sigma \in \tau_{n}(\mu, S)$ for $n=1,2,3,4$. Let $\sigma=\otimes_{v} \sigma_{v} \in \mathcal{T}_{n}(\mu, S)$ and let, for each $v$, the representation

$$
\Sigma_{v}: W_{F_{v}}^{\prime} \rightarrow G L_{n}(\mathbb{C})
$$

parametrize $\sigma_{v}([20,21])$. Let

$$
L\left(s,\left(\pi_{1 v} \otimes \pi_{2 v}\right) \times \sigma_{v}\right)=L\left(s, \varphi_{1 v} \otimes \varphi_{2 v} \otimes \Sigma_{v}\right),
$$

where the $L$-function on the right hand side of (5.3) is that of Artin. Let

$$
L\left(s,\left(\pi_{1} \otimes \pi_{2}\right) \times \sigma\right)=\prod_{v} L\left(s,\left(\pi_{1 v} \otimes \pi_{2 v}\right) \times \sigma_{v}\right) .
$$

The root number $\varepsilon\left(s,\left(\pi_{1} \otimes \pi_{2}\right) \times \sigma\right)$ is defined similarly by

$$
\varepsilon\left(s,\left(\pi_{1} \otimes \pi_{2}\right) \times \sigma\right)=\prod_{v} \varepsilon\left(s, \varphi_{1 v} \otimes \varphi_{2 v} \otimes \Sigma_{v}, \psi_{v}\right),
$$

where $\psi=\otimes_{v} \psi_{v}$ is a non-trivial character of $F \backslash \mathbb{A}_{F}$ and the root number on the right are again those of Artin.

a) $L\left(s,\left(\pi_{1} \otimes \pi_{2}\right) \times \sigma\right)$ is entire,

b) $L\left(s,\left(\pi_{1} \otimes \pi_{2}\right) \times \sigma\right)$ is bounded in vertical strips of finite width,

and

c) $L\left(s,\left(\pi_{1} \nabla \pi_{2}\right) \times \sigma\right)=\varepsilon\left(s,\left(\pi_{1} \otimes \pi_{2}\right) \times \sigma\right) L\left(1-s,\left(\tilde{\pi}_{1} \otimes \tilde{\pi}_{2}\right) \times \tilde{\sigma}\right)$ for all $\sigma \in \mathcal{T}_{n}(\mu, S)$ and $n=1,2,3,4$. Then there exists an automorphic representation $\prod^{\prime}=\otimes_{v} \prod_{v}^{\prime}$ such that $\prod_{v}^{\prime}=\pi_{1 v} \otimes \pi_{2 v}$ for all $v \notin S$.

Conditions 5.6.a), b) and c) are proved by appealing to Langlands-Shahidi method $[19,27,39,48,49,50,51,52]$. For that, for each $n=1,2,3$, 4, we need to choose a triple $(\mathbf{G}, \mathbf{M}, \Pi)$ in which $\mathbf{G}$ is a connected reductive group with $\mathbf{M}$ a Levi subgroup, both defined over $F$. Moreover, $\Pi$ is a cuspidal representation of $M=\mathbf{M}\left(\mathbb{A}_{F}\right)$ defined by $\pi_{1}, \pi_{2}$ and $\sigma$, in such a way that the constant term of the Eisenstein series defined by $G, M$ and $\prod$ has $L^{S}\left(s,\left(\pi_{1} \otimes \pi_{2}\right) \times \sigma\right)$ as the main $L$-function in its constant term [38, 39, 48, 51, 52], where

$$
L^{S}\left(s,\left(\pi_{1} \otimes \pi_{2}\right) \times \sigma\right)=\prod_{v \notin S} L\left(s,\left(\pi_{1 v} \otimes \pi_{2 v}\right) \times \sigma_{v}\right) .
$$


Here is a table for pairs $\left(\mathbf{G}, \mathbf{M}_{D}\right)$ which are used in [34] to give the analytic properties of $L\left(s,\left(\pi_{1} \otimes \pi_{2}\right) \times \sigma\right)$ for each $n, n=1,2,3,4$,

\begin{tabular}{|l|l|l|}
\hline$n$ & \multicolumn{1}{|}{$\mathbf{G}$} & \multicolumn{1}{c|}{$\mathbf{M}_{D}$} \\
\hline 1 & $S L_{5}$ & $S L_{2} \times S L_{3}$ \\
\hline 2 & $D_{5}^{s c}$ & $S L_{2} \times S L_{3} \times S L_{2}$ \\
\hline 3 & $E_{6}^{s c}$ & $S L_{2} \times S L_{3} \times S L_{3}$ \\
\hline 4 & $E_{7}^{s c}$ & $S L_{2} \times S L_{3} \times S L_{4}$ \\
\hline
\end{tabular}

where " $s c$ " denotes the simply connected member of the corresponding isogeny class of $\mathbf{G}_{D}$, and $\mathbf{M}_{D}$ and $\mathbf{G}_{D}$ are the derived groups of $\mathbf{M}$ and $\mathbf{G}$, respectively.

Applying the techniques of the method $[19,27,48,49,50,51,52]$ we get the following theorem. More precisely, we obtain 6.5a) from Kim's observation [27] (Proposition 2.1 of [34]), 5.6b) from Gelbart-Shahidi [19] and 5.6c) and corresponding multiplicativity results from $[48,49,50,51,52]$.

(5.8) Theorem [34]. Conditions 5.6a), b) and c) are valid and therefore there exists an automorphic representation $\prod^{\prime}=\otimes_{v} \prod_{v}^{\prime}$ of $G L_{6}\left(\mathbb{A}_{F}\right)$ such that $\prod_{v}^{\prime}=$ $\pi_{1 v} \otimes \pi_{2 v}$ for $\forall v \notin S$.

To prove that $\prod_{v}^{\prime}=\pi_{1 v} \otimes \pi_{2 v}$ for all $v$, more work was needed. In particular, one needs to use base change, both normal and non-normal cubic [3, 37] , as well as a result from theory of $K$-types [7] which is an appendix to [34]. As a bonus one gets the equality of triple product $L$-functions $L\left(s, \pi_{1 v} \times \pi_{2 v} \times \sigma_{v}\right)$ and root numbers $\varepsilon\left(s, \pi_{1 v} \times \pi_{2 v} \times \sigma_{v}, \psi_{v}\right)$ defined by Langlands-Shahidi method [52] with Artin factors for all $v$. Note that $L\left(s, \pi_{1 v} \times \pi_{2 v} \times \sigma_{v}\right)$ and $\varepsilon\left(s, \pi_{1 v} \times \pi_{2 v} \times \sigma_{v}, \psi_{v}\right)$ are defined completely by methods of harmonic analysis (cf. [49, 52]) while the Artin $L$-functions $L\left(s, \varphi_{1 v} \otimes \varphi_{2 v} \otimes \Sigma_{v}\right)$ and root numbers $\varepsilon\left(s, \varphi_{1 v} \otimes \varphi_{2 v} \otimes \Sigma_{v}, \psi_{v}\right)$ are completely of arithmetic nature [37] and their equality is quite deep. Note that $L\left(s, \pi_{1 v} \times \pi_{2 v} \times \sigma_{v}\right)=L\left(s, \varphi_{1 v} \otimes \varphi_{2 v} \otimes \Sigma_{v}\right)=L\left(s,\left(\pi_{1 v} \otimes \pi_{2 v}\right) \times \sigma_{v}\right)$ for all $v \notin S$, trivially.

(5.9) Remark. Suppose $\mathrm{Sym}^{3} \pi$ is not cuspidal. Then either $\pi$ is monomial in which case $\mathrm{Sym}^{2} \pi$ is not cuspidal, or $\mathrm{Sym}^{2} \pi$ is cuspidal, but there exists a grössencharacter $\eta \neq 1$ such that

$$
\mathrm{Sym}^{2} \pi \cong \mathrm{Sym}^{2} \pi \otimes \eta .
$$

Then $\eta^{3}=1$ and $\eta$ determines a cubic cyclic extension $E / F$. It is quite easy to show that then $\pi_{E}$, the base change of $\pi$, is monomial (cf. [53]). Let $\sigma_{E}$ be the dihedral representation of $W_{E}$ such that $\pi_{E}=\pi\left(\sigma_{E}\right)$. Then, as it is explained in Lemma 6.5 of [34], $\sigma_{E}$ can be extended to a two dimensional representation of $W_{F}$ which is of tetrahedral type (cf. $\left.[16,34,37]\right)$. This is possible since $\sigma_{E}$ is invariant under $\operatorname{Gal}(E / F)$ and $H^{2}\left(W_{F}, \mathbb{C}^{*}\right)=1$. The fact that representation $\sigma$ is of tetrahedral type follows from the fact that $\pi$ is not monomial, together with an inspection of the image of $\sigma$ in $P G L_{2}(\mathbb{C})$ to rule out the octahedral and 
icosahedral cases, since it must contain a non-trivial normal subgroup of index 3 (cf. the diagram in page 198 of [16]). It then follows that $\pi(\sigma)_{E}=\pi\left(\sigma_{E}\right)=\pi_{E}$ and therefore (cf. [2]) $\pi(\sigma)=\pi \otimes \eta^{i}, i=0,1,2$, where $\pi(\sigma)$ is the cuspidal representation of $G L_{2}\left(\mathbb{A}_{F}\right)$ attached to $\sigma$ by Langlands [37]. Twisting $\sigma$ by $\eta^{-i}$, if necessary, implies that there exists a tetrahedral representation $\sigma$ such that $\pi=\pi(\sigma)$. Theorem 6.1 of [34] then can be formulated as:

(5.11) Proposition. Suppose $\pi$ is a cuspidal representation of $G L_{2}\left(\mathbb{A}_{F}\right)$. Then the following statements are equivalent:

a) $\mathrm{Sym}^{2} \pi$ is cuspidal, but $\mathrm{Sym}^{3} \pi$ is not.

b) $\mathrm{Sym}^{2} \pi$ is cuspidal and (5.10) holds, i.e., there exists a non-trivial cubic grössencharacter $\eta$ of $F$ such that

$$
\mathrm{Sym}^{2} \pi \cong \mathrm{Sym}^{2} \pi \otimes \eta \text {. }
$$

c) $\pi$ is of tetrahedral type, i.e., there exists a tetrahedral representation $\sigma$ of $W_{F}$, an irreducible two dimensional complex representation of $W_{F}$ whose image in $P G L_{2}(\mathbb{C})$ is isomorphic to $A_{4}$, such that $\pi=\pi(\sigma)$ through the Langlands correspondence.

In particular, if $\pi$ corresponds to a holomorphic cusp form, then $\sigma$ is odd, i.e., $\operatorname{det}(\sigma(c))=-1$, where $c$ is the image of complex conjugation in $\operatorname{Gal}(\overline{\mathbb{Q}} / \mathbb{Q})$.

Similar results concerning cuspidality of $\mathrm{Sym}^{4} \pi$ and its connection with octahedral representations of Galois group are established in [33].

\section{Functoriality of Higher Symmetric Powers and Kac-Moody GROUPS}

This section is quite speculative and the reader must not look for much rigor, but rather philosophical remarks.

In this section we speculate on whether a straightforward generalization of the theory of Eisenstein series to Kac-Moody groups leads to new triple product $L$-functions and thus cases of functoriality. It becomes evident that a straightforward generalization will lead to nothing new and one needs a conceptually different approach if one hopes to generalize the Langlands-Shahidi method [19, $27,39,49,50,51,52]$ to these groups. We start by discussing the existence of $\mathrm{Sym}^{5} \pi$.

An application of Clebsch-Gordan formula implies that the functoriality of

$$
\operatorname{Sym}^{5}: G L_{2}(\mathbb{C}) \rightarrow G L_{6}(\mathbb{C})
$$

follows immediately from that of

$$
\rho_{2} \otimes \rho_{5}: G L_{2}(\mathbb{C}) \times G L_{5}(\mathbb{C}) \rightarrow G L_{10}(\mathbb{C}) .
$$


To apply the converse theorem we discussed earlier [11], we need to have a way of establishing analytic properties of triple product $L$-functions on $G L_{2} \times G L_{5} \times$ $G L_{n}, n=1,2,3, \ldots, 8$. To use the Langlands-Shahidi method we need to consider a group $\mathbf{G}$, possibly infinite dimensional, with a Levi subgroup $\mathbf{M}$ whose derived group is $\mathbf{M}_{D}=S L_{2} \times S L_{5} \times S L_{n}, n=1,2, \ldots, 8$.

In the notation of [26], $\mathbf{G}$ will be a Kac-Moody group whose Cartan matrix will be of type $T_{2,5, n}$. (We will assume the existence of these groups as well.) When $n=1,2,3$, one can take $\mathbf{G}=S L_{7}, D_{7}^{s c}, E_{8}$. In these cases $\mathbf{G}$ is finite dimensional and the method applies and should give all the necessary analytic properties of $L\left(s, \pi_{1} \times \pi_{2} \times \sigma\right)$, where $\pi_{1}, \pi_{2}$ and $\sigma$ are cuspidal representations of $G L_{2}\left(\mathbb{A}_{F}\right), G L_{5}\left(\mathbb{A}_{F}\right)$ and $G L_{n}\left(\mathbb{A}_{F}\right), n=1,2,3$, respectively.

Now assume $n \geq 4$. None of the groups $T_{2,5, n}$ is now finite dimensional. In fact, they are not even affine (loop groups) and it is only $T_{2,5,4}$ which is hyperbolic, i.e., its proper connected subdiagrams are either finite dimensional or affine.

The problem is that when $n \geq 4$, none of the Levi subgroups $\mathbf{M}$ are self associate as we explain below.

(6.1) Definition. Let $\mathbf{P}=\mathbf{M N}$ be a maximal parabolic subgroup of a Kac-Moody group $\mathbf{G}$. Assume $\mathbf{M} \supset \mathbf{T}$, a maximal torus whose restricted simple roots $\Delta$ generate a minimal parabolic subgroup contained in $\mathbf{P}$. Let $\theta \subset \Delta$ generate $\mathbf{M}$. Since $\mathbf{P}$ is maximal $\Delta \backslash \theta=\{\alpha\}$. The Levi subgroup $\mathbf{M}$ or equivalently $\theta$ is called self-associate, if there exists an element $w_{0}$ in the Weyl group $W\left(\mathbf{A}_{0}, \mathbf{G}\right)$ of the maximal split subtorus $\mathbf{A}_{0}$ of $\mathbf{T}$ such that $w_{0}(\theta)=\theta$ and $w_{0}(\alpha)<0$.

(6.2) Lemma. Assume $\operatorname{dim} \mathbf{G}=\infty$, but $\operatorname{dim} \mathbf{M}<\infty$. Then $\mathbf{M}$ cannot be selfassociate.

Proof. Suppose $\mathbf{M}$ is self-associate. Since $\mathbf{M}$ is finite dimensional, the Weyl group of $\mathbf{A}_{0}$ in $\mathbf{M}$ has a longest element $w_{0}^{\mathbf{M}}$. Then $w_{0} w_{0}^{\mathbf{M}}(\theta)=-\theta$ and $w_{0} w_{0}^{\mathbf{M}}(\alpha)<0$. Thus $w_{0} w_{0}^{\mathbf{M}}$ must in particular send positive imaginary roots to negative roots which is a contradiction (Proposition 5.2 of [26]; also see Remark 5.9 of [26]). We recall that a root $\alpha$ is imaginary if and only if there is no $w \in W\left(\mathbf{A}_{0}, \mathbf{G}\right)$ such that $w(\alpha)$ is simple.

This observation can be experimentally validated by calculating, formally and under all the simplifying assumptions, the constant term of the Eisenstein series attached to $(\mathbf{G}, \mathbf{M})$ in the cases $n=4, \ldots, 8$ discussed earlier, following Garland $[14,15]$ who generalizes Langlands $[39,40]$. One can see that in each case all that remains is the trivial term $f(e)$. In fact, given any Weyl group element $w \neq 1$, one can always find a subset of roots with root spaces in $\mathbf{N}$ which when conjugated by $w$ generate the unipotent radical $\mathbf{N}_{1}$ of a proper parabolic subgroup in $\mathbf{M}$. One then needs to proceed with the same type of argument as in [39] and integrate the cusp form on $M=\mathbf{M}\left(\mathbb{A}_{F}\right)$ over $\mathbf{N}_{1}(F) \backslash \mathbf{N}_{1}\left(\mathbb{A}_{F}\right)$ which evidently vanishes. 
Although we have only checked this carefully in the example $E_{6}^{(1)}$ below, the result seems to be quite general. This is very different from the finite dimensional cases where one at least always gets the non-trivial term, i.e., the one involving the quotient of product of $L$-functions.

(6.3) Conclusion. The straightforward generalization of the theory of Eisenstein series to infinite dimensional cases as pursued by Garland [14, 15] will not lead to any new $L$-functions beyond those coming from finite dimensional cases.

On the other hand there are many self-associate non-maximal parabolic subgroups of even loop groups. Our observation then hints that the analytic continuation of Eisenstein series on Kac-Moody groups should reduce to the cases of finite dimensional groups. This ought to be quite useful to Garland who has already started studying them in the case of minimal parabolic subgroups in a systematic way, benefiting from properties of Riemann zeta function [14, 15].

On the dual side, one can try to imitate the situation of finite dimensional groups in the setting of $L$-groups, trying to determine what representations appear in the adjoint action of ${ }^{L} M$, the $L$-group of $M$, on ${ }^{L} \mathfrak{n}$, the Lie algebra of $L$-group of $N$. Of course, we will be interested in the situation where $\operatorname{dim} \mathbf{M}<\infty$, but $\operatorname{dim} \mathbf{N}=\infty$. Many interesting representations do appear. In the example below we will consider the affine group $E_{6}^{(1)}$

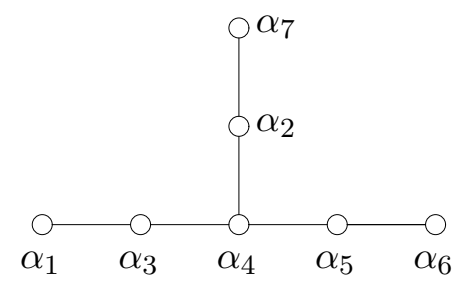

and consider the case where $\alpha=\alpha_{4}, \theta=\left\{\alpha_{1}, \alpha_{3}, \alpha_{7}, \alpha_{2}, \alpha_{5}, \alpha_{6}\right\}$. Instead, we will calculate $\operatorname{ad}\left(\mathfrak{m}_{\mathbb{C}}\right)$ on $\mathfrak{n}_{\mathbb{C}}$, the complex Lie algebra of roots restricting to $\alpha=\alpha_{4}$ and its multiples, where $\mathfrak{m}_{\mathbb{C}}$ is the Levi subalgebra of $\mathfrak{g}_{\mathbb{C}}$, the complex affine Lie algebra of type $E_{6}^{(1)}$, generated by $\theta$. Using the usual calculations by means of Cartan matrices and our knowledge of highest weights it is easy to see that the following representations

$$
\begin{aligned}
& r_{1}=\delta_{1}+\delta_{7}+\delta_{6}, \\
& r_{2}=\delta_{3}+\delta_{2}+\delta_{5}
\end{aligned}
$$

and

$$
r_{3}=\left(\delta_{1}+\delta_{3}\right) \oplus\left(\delta_{2}+\delta_{7}\right) \oplus\left(\delta_{5}+\delta_{6}\right)
$$

appear in this action, each infinitely many times. We note that

$$
\mathfrak{m}_{\mathbb{C}}=\operatorname{sl}_{3}(\mathbb{C}) \oplus \operatorname{sl}_{3}(\mathbb{C}) \oplus \operatorname{sl}_{3}(\mathbb{C})
$$


and that $\delta_{i}$ 's denote the fundamental weights attached to $\alpha_{i}$ 's as in $[39,51]$.

These representations are quite interesting. In fact, $r_{1}$ is the triple tensor product of standard representations of $s l_{3}(\mathbb{C})$ 's and $r_{2}=\tilde{r}_{1}$. The representation $r_{3}$ is the direct sum of three 8-dimensional adjoint representations $\delta_{1}+\delta_{3}, \delta_{2}+\delta_{7}$ and $\delta_{5}+\delta_{6}$ of these $s l_{3}(\mathbb{C})$ 's. In contrast to finite dimensional theory, we notice that it is now possible to have both a representation and its contragredient appear in the same adjoint action. It is also interesting to notice that it is now possible to have a representation $r_{i}$ which is reducible. The imaginary roots play a crucial role [26]. Neither happens in the case of finite dimensional groups [39, 51]. Clearly $r_{1}$ and $r_{2}$ can define triple product $L$-functions which are new. The same is true for $r_{3}$ as it is quite hard to get ones hands on adjoint $L$-functions.

The irreducible constituents of $r_{3}$ are equivalent as representations of $s l_{3}(\mathbb{C})$. They each appear as an irreducible component of the adjoint action of $s l_{3}(\mathbb{C})$ as a Levi subalgebra of the complex affine Lie algebra $A_{2}^{(1)}$, generated say by $\left\{\alpha_{1}, \alpha_{2}\right\}$, on the subspace of roots restricting to $\alpha_{3}$ and its multiples, appearing infinitely many times. In fact, one can show that for any positive integer $n$, the pair $\left\{A_{n}^{(1)}, s l_{n+1}(\mathbb{C})\right\}$ will have the adjoint representation of $s l_{n+1}(\mathbb{C})$ (on itself) appearing infinitely many times, when $s l_{n+1}(\mathbb{C})$ is the Levi subalgebra generated, for example, by $\left\{\alpha_{1}, \ldots, \alpha_{n}\right\} \subset\left\{\alpha_{1}, \ldots, \alpha_{n+1}\right\}$.

The fact that both $r_{1}$ and $\tilde{r}_{1}=r_{2}$ appear in the adjoint action in the $E_{6}^{(1)}$ case may not be in disagreement with the earlier observation on triviality of the constant term. In fact, even if the constant term were to imply the meromorphy of

$$
L\left(s, \pi, r_{1}\right) L\left(2 s, \tilde{\pi}, r_{1}\right) / L\left(1+s, \pi, r_{1}\right) L\left(1+2 s, \tilde{\pi}, r_{1}\right)
$$

as in finite dimensional cases, taking into account the possible meromorphy for $L\left(3 s, \pi, r_{3}\right) / L\left(1+3 s, \pi, r_{3}\right)$ coming from $A_{2}^{(1)}$, discussed before, it will be awfully hard to prove the meromorphy of individual $L$-function $L\left(s, \pi, r_{1}\right)$.

To wit, let us point out that Langlands [39] discussed the meromorphic continuation of $L\left(s, \pi, r_{1}\right)$ in the finite dimensional cases, by assuming the meromorphy for all other $L\left(s, \pi, r_{1}\right), i \geq 2$. It was in $[51,52]$ where we first proved the necessary induction in general to make his argument self contained. This meant showing that each $L\left(s, \pi, r_{i}\right), i \geq 2$, already appears in another setting as a first $L$-function. It is quite evident that this is not the case even in the example of $E_{6}^{(1)}$, when one deals with infinite dimensional group.

In fact, unfortunately, a direct induction argument starting with a line of absolute convergence for $L\left(s, \pi, r_{1}\right)$, applied to (6.7), only implies the meromorphy of $L\left(s, \pi, r_{1}\right)$ for $\operatorname{Re}(s)>0$. On the other hand, if one assumes what we usually 
call the "crude functional equation" $[49,52]$

$$
L\left(s, \pi, r_{1}\right) L\left(2 s, \tilde{\pi}, r_{1}\right)=C(s, \pi) L\left(1-s, \tilde{\pi}, r_{1}\right) L\left(1-2 s, \pi, r_{1}\right)
$$

with a meromorphic function $C(s, \pi)$, one concludes that

$$
L\left(1-s, \tilde{\pi}, r_{1}\right) L\left(1-2 s, \pi, r_{1}\right) / L\left(1+s, \pi, r_{1}\right) L\left(1+2 s, \tilde{\pi}, r_{1}\right)
$$

is meromorphic on all of $\mathbb{C}$. ¿From this one can prove, using another not so obvious induction, that $L\left(s, \pi, r_{1}\right)$ is meromorphic on all of $\mathbb{C}$.

(The complex variable $s$ is injected into the Eisenstein series through $s \tilde{\alpha}$, where $\tilde{\alpha}$ is an element in the dual of $\hat{\mathfrak{h}}^{e}$ in the notation of [14], defined by $\langle\tilde{\alpha}, \beta\rangle=1$ for $\beta=\alpha_{4}$ and $\langle\tilde{\alpha}, \beta\rangle=0$ for $\beta \in\left\{\alpha_{1}, \alpha_{3}, \alpha_{7}, \alpha_{2}, \alpha_{5}, \alpha_{6}\right\}$. This replaces $\rho /\langle\rho, \alpha\rangle$ (cf. [51]) of the finite dimensional cases, avoiding $\rho$, the half sum of roots in $\mathbf{N}$, which has no meaning in the infinite dimensional setting.)

The reader must now appreciate that even assuming that appropriate quotients of products of $L$-functions are meromorphic as in the finite dimensional cases, it is much harder to prove the meromorphy of individual $L$-functions and in general new inputs such as the existence of a crude functional equation may be necessary.

Taking different $T_{p, q, r}$ one can see that for every triple of positive integers $(p, q, r)$, the triple tensor product representation of $s l_{p}(\mathbb{C}) \oplus s l_{q}(\mathbb{C}) \oplus s l_{r}(\mathbb{C})$ appears in the corresponding adjoint action.

The main question is whether it is possible to detect these complex representations in a dual way in the representation theory of an infinite dimensional group of type $T_{p, q, r}$, say over a number field.

As explained earlier, a straightforward generalization of the theory of Eisenstein series from finite dimensional groups to infinite dimensional ones as pursued by Garland $[14,15]$ would not suffice.

Considering the complicated nature of these groups, including the lack of a Haar measure, it may be possible that to understand their spectrum one may need other tools beside the Eisenstein series. That remains to be seen, but there may be some hope in reaching some insight by looking at the representation theory of these groups over local fields as now being pioneered by Gaitsgory and Kazhdan [13]. Finally we refer to Kim and Lee [31] for a first step towards the notion of Satake parameters for loop groups. 


\section{Higher Symmetric Power $L-$ Functions}

Although we cannot prove the functoriality of $\mathrm{Sym}^{m} \pi$ for $m \geq 5$, using automorphy of $\mathrm{Sym}^{3} \pi$ and $\mathrm{Sym}^{4} \pi$, we can prove certain analytic properties of

$$
L^{S}\left(s, \pi, \operatorname{Sym}^{m} \otimes \chi\right)=\prod_{v \notin S} \prod_{j=0}^{m}\left(1-\alpha_{v}^{j} \beta_{v}^{m-j} \chi_{v}\left(\varpi_{v}\right) q_{v}^{-s}\right)^{-1},
$$

for $m \leq 8$, where $\pi=\otimes_{v} \pi_{v}$ with $\pi_{v}$ parametrized by $t_{v}=\operatorname{diag}\left(\alpha_{v}, \beta_{v}\right) \in G L_{2}(\mathbb{C})$ for all $v \notin S$ and $\chi=\otimes_{v} \chi_{v}$ is an idele class character which is unramified for all $v \notin S$. This is the subject matter of a collection of results proved in [33]. The following result follows from the absolute convergence of Rankin product $L$-functions for $G L_{n}\left(\mathbb{A}_{F}\right) \times G L_{m}\left(\mathbb{A}_{F}\right)$ established by Jacquet and Shalika for $R e(s)>1$ in [25], and the existence of $\operatorname{Sym}^{3} \pi$ and $\mathrm{Sym}^{4} \pi$. It has not appeared elsewhere and seems to be new.

(7.2) Theorem (Kim-Shahidi). The partial $L$-functions $L^{S}\left(s, \pi, \mathrm{Sym}^{m} \otimes \chi\right)$ are all absolutely convergent for $\operatorname{Re}(s)>1$ for all $m \leq 8$ and any idele class character $\chi$ of $\mathbb{A}_{F}^{*}$, unramified outside $S$.

Proof. Each of the $L$-functions $L^{S}\left(s, \pi, \operatorname{Sym}^{m} \otimes \chi\right)$ can be written as a ratio of an appropriate $L^{S}\left(s, \operatorname{Sym}^{p} \pi \times\left(\operatorname{Sym}^{q} \pi \otimes \chi\right)\right)$ with $2 \leq p, q \leq 4$, with a product of $L^{S}\left(s, \pi, \operatorname{Sym}^{r} \otimes \omega_{\pi}^{t} \chi\right)$ with $r<m$ and some integer $t$ (cf. proofs of Propositions 4.2-4.6 of [33]). It follows from the main result of [25] that each

$$
L^{S}\left(s, \operatorname{Sym}^{p} \pi \times\left(\operatorname{Sym}^{q} \pi \otimes \chi\right)\right)
$$

is absolutely convergent if $p$ and $q \leq 4$. Now the theorem is proved by appealing to its validity for smaller $m$ inductively.

It was suggested by Langlands in [38] that if one knows the absolute convergence of $L^{S}\left(s, \pi, \operatorname{Sym}^{m}\right)$ for $\operatorname{Re}(s)>1$ and for all $m$, then one can immediately conclude the Ramanujan-Petersson's conjecture $\left|\alpha_{v}\right|=\left|\beta_{v}\right|=1$. (In fact, $\operatorname{Re}(s) \geq \sigma_{0}$ for some $\sigma_{0}$ independent of $m$ would suffice.)

The following Corollary of our theorem gives an example of the kind of estimates that one can get if one only knows the convergence for some of these $L$-functions. Although it is weaker than estimates (3.5) and (3.6), it is the first instant that Langlands suggestion is partially used as finally there are enough symmetric power $L$-functions to use to show the following corollary.

(7.3) Corollary. $q_{v}^{-1 / 8} \leq\left|\alpha_{v}\right|,\left|\beta_{v}\right| \leq q_{v}^{1 / 8}$.

The following corollary is crucial in the proof of (3.6) by Kim and Sarnak [32]. It is proved here using our Theorem 7.2.

(7.4) Corollary. The L-function $L^{S}\left(s, \mathrm{Sym}^{4} \pi, \mathrm{Sym}^{2}\right)$ is absolutely convergent for $\operatorname{Re}(s)>1$. 
Proof. Since

$$
L^{S}\left(s, \operatorname{Sym}^{4} \pi \times \operatorname{Sym}^{4} \pi\right)=L^{S}\left(s, \operatorname{Sym}^{4} \pi, \operatorname{Sym}^{2}\right) L^{S}\left(s, \operatorname{Sym}^{4} \pi, \Lambda^{2}\right)
$$

is absolutely convergent for $\operatorname{Re}(s)>1$, the assertion follows if we prove it for $L^{S}\left(s, \operatorname{Sym}^{4} \pi, \Lambda^{2}\right)$. It is easy to show

$$
L^{S}\left(s, \operatorname{Sym}^{4} \pi, \Lambda^{2}\right)=L^{S}\left(s, \pi, \operatorname{Sym}^{6} \otimes \omega_{\pi}\right) L^{S}\left(s, \pi, \operatorname{Sym}^{2} \otimes \omega_{\pi}^{3}\right) .
$$

The absolute convergence of the left hand side of (7.5) now follows from Theorem 7.2 .

\section{REFERENCES}

[1] J. Arthur, The principle of functoriality, Bull. Amer. Math. Soc. (N.S.) 40, (2002), no. 1, 39-53; Mathematical Challenges of the 21st century (Los Angeles, CA, 2000).

[2] J. Arthur and L. Clozel, Simple Algebras, Base Change, and the Advanced Theory of the Trace Formula, Ann. of Math. Studies 120, Princeton Univ. Press, Princeton, NJ, 1989.

[3] M. Asgari and F. Shahidi, Generic transfer for general spin groups, Duke Math. J. (to appear).

[4] M. Asgari and F. Shahidi, Generic transfer form GSp(4) to GL(4), preprint.

[5] A. Borel, Automorphic L-functions, Proc. Sympos. Pure Math. 33, Part 2 (1979), 27-61.

[6] D. Bump, W. Duke, J. Hoffstein, and H. Iwaniec, An estimate for the Hecke eigenvalues of Maass forms, IMRN 4 (1992), 75-81.

[7] C. J. Bushnell and G. Henniart, On certain dyadic representations (Appendix to [34]), Ann. of Math. 155 (2002), 883-893.

[8] J.W. Cogdell, H.H. Kim, I.I. Piatetski-Shapiro and F. Shahidi, On lifting from classical groups to $G L_{N}$, Publ. Math. Inst. Hautes Études Sci. 93 (2001), 5-30.

[9] J.W. Cogdell, H.H. Kim, I.I. Piatetski-Shapiro and F. Shahidi, Functoriality for the classical groups, Publ. Math. Inst. Hautes Études Sci. 99 (2004), 163-233.

[10] J.W. Cogdell and I.I. Piatetski-Shapiro, Converse theorems for $G L_{n} I$, Publ. Math. Inst. Hautes Études Sci. 79 (1994), 157-214.

[11] J.W. Cogdell and I.I. Piatetski-Shapiro, Converse theorems for GL $L_{n}$ II, J. Reine Angew. Math. 507 (1999), 165-188.

[12] W. Duke and H. Iwaniec, Estimates for coefficients of L-functions, I, Automorphic forms and analytic number theory, (Montreal, PQ, 1989), Univ. Montréal, Montréal, QC, 1990, 43-47.

[13] D. Gaitsgory and D. Kazhdan, Representations of algebraic groups over a 2-dimensional local field, preprint.

[14] H. Garland, Certain Eisenstein series on loop groups: convergence and the constant term, Proceedings of the International Conference on Algebraic Groups and Arithmetic (in honor of M.S. Raghunathan), December 2001, Tata Institute of Fundamental Research, Mumbai, India, pp. 275-319.

[15] H. Garland, Absolute convergence of Eisenstein series on loop groups, preprint.

[16] S. Gelbart, Three lectures on the modularity of $\bar{\rho}_{E, 3}$ and the Langlands reciprocity conjecture, Modular Forms and Fermat's Last Theorem, Springer-Verlag, New York, 1997, pp. 155207.

[17] S. Gelbart, Automorphic forms on adéle groups, Princeton University Press, Princeton, NJ, 1975, Annals of Mathematics Studies, no. 83.

[18] S. Gelbart and H. Jacquet, A relation between automorphic representations of $G L(2)$ and GL(3), Ann. Sci. École Norm. Sup. 11 (1978); no. 4, 471-542. 
[19] S. Gelbart and F. Shahidi, Boundedness of automorphic L-functions in vertical strips, J. Amer. Math. Soc. 14 (2001); no. 1, 79-107.

[20] M. Harris and R. Taylor, The geometry and cohomology of some simple Shimura varieties, Annals of Mathematics Studies, Vol. 151, Princeton University Press, Princeton, NJ, 2001.

[21] G. Henniart, Une preuve simple des conjectures de Langlands pour $G L(n)$ sur un corps p-adique, Invent. Math. 139 (2000), 439-455.

[22] G. Henniart, La conjecture de Langlands locale pour $G L(3)$, Mémoires de la Soc. Math. de France 11/12 (1984), 1-186.

[23] R. Howe and I.I. Piatetski-Shapiro A counter-example to the "generalized Ramanujan conjecture" for (quasi)-split groups, Proc. Sympos. Pure Math. 33; Part 1 (1979), 315-322.

[24] H. Jacquet, I.I. Piatetski-Shapiro, and J.A. Shalika, Relévement cubique non normal, C.R. Acad. Sci Paris Sér. I Math. 292 (1981), 567-571.

[25] H. Jacquet and J.A. Shalika, On Euler products and the classification of automorphic representations, I, II, Amer. J. Math. 103 (1981); 499-558 and 777-815.

[26] V.G. Kac, Infinite dimensional Lie algebras, Cambridge University Press, Cambridge-New York-Melbourne, 1995.

[27] H.H. Kim, Langlands-Shahidi method and poles of automorphic Lfunctions: application to exterior square L-functions, Canad. J. Math. 51 (1999); no. 4, 835-849.

[28] H.H. Kim, Functoriality for the exterior square of $G L_{4}$ and the symmetric fourth of $G L_{2}$; with appendix 1 by D. Ramakrishnan and appendix 2 by H.H. Kim and P. Sarnak, J. Amer. Math. Soc. 16 (2002); no. 1, 139-183.

[29] H.H. Kim, On local L-functions and normalized intertwining operators, Canad. J. Math. 57 (2005), 535-597.

[30] H.H. Kim and M. Krishnamurthy, Base change lift from unitary groups to $G L_{N}$, IMRP, 2005; no. 1 (2005), 1-52.

[31] H.H. Kim and K-H. Lee, Spherical Hecke algebras of $\mathrm{SL}_{2}$ over 2dimensional fields, American J. Math. 126 (2004), 1381-1399.

[32] H.H. Kim and P. Sarnak, Refined estimates towards the Ramanujan and Selberg conjectures, Appendix 2 to [46], J. Amer. Math. Soc. 16 (2002); no. 1, 175-181.

[33] H.H. Kim and F. Shahidi, Cuspidality of symmetric powers with applications, Duke Math. J. 112 (2002); no. 1, 177-197.

[34] H.H. Kim and F. Shahidi, Functorial products for $G L_{2} \times G L_{3}$ and the symmetric cube for $G L_{2}$, with an appendix by C.J. Bushnell and G. Henniart, Ann. of Math. 155 (2002); no. 3, 837-893.

[35] N. Kurokawa, Examples of eigenvalues of Hecke operators on Siegel cusp forms of degree two, Invent. Math. 49 (1978); 149-165.

[36] P. Kutzko, The Langlands conjecture for $G L_{2}$ of a local field, Ann. of Math. 112 (1980), 381-412.

[37] R.P. Langlands, Base Change for GL(2), Ann. of Math. Studies, vol. 96, Princeton University Press, Princeton, NJ, 1980.

[38] R.P. Langlands, Problems in the theory of automorphic forms, in Lecture Notes in Math. 170, Springer-Verlag, Berlin-Heidelberg-New York, 1970, 18-86.

[39] R.P. Langlands, Euler products, Yale Mathematical Monographs, 1, Yale University Press, New Haven, CT, 1971.

[40] R.P. Langlands, On the functional equations satisfied by Eisenstein series, Lecture Notes in Mathematics, Vol. 544, Springer-Verlag, Berlin, 1976.

[41] R.P. Langlands, On the classification of irreducible representations of real algebraic groups, Representation Theory and Harmonic Analysis on Semisimple Lie Groups, Math. Surveys Monogr., Vol. 31, Amer. Math. Soc., Providence, RI, 1989, pp. 101-170. 
[42] W. Luo, Z. Rudnick and P. Sarnak, On the generalized Ramanujan conjecture for GL $(n)$, Automorphic forms, automorphic representations, and arithmetic, Proc. Sympos. Pure Math. 66, 1999, 301-310.

[43] I.I. Piatetski-Shapiro, Multiplicity one theorems, Proc. Sympos. Pure Math. 33, Part 1 (1979), 209-212.

[44] D. Ramakrishnan, Modularity of the Rankin-Selberg L-series, and multiplicity one for $S L(2)$, Ann. of Math. 152 (2000), 45-111.

[45] P. Sarnak, Spectra of hyperbolic surfaces, Bull. Amer. Math. Soc. (N.S.) 40 (2003), 441-478.

[46] I. Satake, Spherical functions and Ramanujan Conjecture, Proc. Sympos. Pure Math. 9 (1966), 258-264.

[47] A. Selberg, On the estimation of Fourier coefficients of modular forms, Proc. Sympos. Pure Math. 8 (1965), 1-15.

[48] F. Shahidi, Fourier transforms of intertwining operators and Plancherel measures for $G L(n)$, Amer. J. Math. 106 (1984), 67-111.

[49] F. Shahidi, On certain L-functions, Amer. J. Math. 103 (1981); no. 2, 297-355.

[50] F. Shahidi, Local coefficients as Artin factors for real groups, Duke Math. J. 52, 1985, no. 4, 973-1007.

[51] F. Shahidi, On the Ramanujan conjecture and finiteness of poles for certain L-functions, Ann. of Math. 127 (1988); no. 3, 547-584.

[52] F. Shahidi, A proof of Langlands' conjecture on Plancherel measures; complementary series for $p$-adic groups, Ann. of Math. 132 (1990); no. 2, 273-330.

[53] F. Shahidi, Symmetric power L-functions for $G L(2)$, Elliptic curves and related topics, with an appendix by J-P. Serre, CRM Proc. Lecture Notes, vol. 4, Amer. Math. Soc., Providence, RI, 1994, pp. 159-182.

[54] F. Shahidi, Automorphic L-functions and functoriality, Proceedings of the International Congress of Mathematicians, Vol. II (Beijing, 2002), Higher Ed. Press, Beijing, 2002, pp. $655-666$.

[55] F. Shahidi, Functoriality and small eigenvalues of Laplacian on Riemann surfaces, Surveys in Differential Geometry, International Press (to appear).

[56] F. Shahidi, On the Ramanujan conjecture for quasisplit groups, Asian J. Math. 8 (2004), 813-836.

[57] J.A. Shalika, The multiplicity one theorem for $G L(n)$, Ann. of Math. 100 (1974), 171-193.

Freydoon Shahidi

Purdue University

West Lafayette, IN 47907, U.S.A.

E-mail: shahidi@math.purdue.edu 